\title{
Asynchronous Uplink Non-orthogonal Multiple Access (NOMA) with Cyclic Prefix
}

\author{
Xiaolu Wang ${ }^{\dagger}$, Fabrice Labeau ${ }^{\ddagger}$, Lin $\mathrm{Mei}^{\dagger}$ \\ ${ }^{\dagger}$ Communication Research Center, Harbin Institute of Technology, Harbin, Heilongjiang, China \\ ${ }^{\ddagger}$ Electrical and Computer Engineering, McGill University, Montreal, QC, Canada \\ Email: xiaolu_wang@outlook.com, fabrice.labeau@mcgill.ca, meilin@hit.edu.cn
}

\begin{abstract}
Non-orthogonal multiple access (NOMA) has been recognized as a promising technique for future radio access. In this paper, different from [3], it is proposed that the cyclic prefix (CP) is used in the asynchronous uplink NOMA transmission for mitigating asynchronous effects. Through analyzing the interuser interference (IUI) and residual interference, it is shown that the asynchronism can degrade the uplink NOMA users' bit error rate (BER) performance severely for the conventional-successive interference cancellation (Conv-SIC) technique. By applying the SIC-phase compensation (SIC-PC) technique and CP, the asynchronous effects can be mitigated. The BER performance of near and far users can be improved substantially, compared with other existing successive interference cancellation (SIC) techniques.
\end{abstract}

\section{INTRODUCTION}

Orthogonal multiple access (OMA) has been adopted as a main common technology in IEEE 802.16e-based Worldwide Interoperability for Microwave Access (WiMAX) and Long Term Evolution (LTE) standards [1]. In LTE, Orthogonal frequency division multiple access (OFDMA) is used in the downlink and single carrier-frequency division multiple access (SC-FDMA) in the uplink, while WiMAX adopts OFDMA in both the uplink and the downlink. A cyclic prefix (CP) is appended to the beginning of an OMA symbol and discarded at the receiver to provide the immunity against inter-symbol interference (ISI) and inter-carrier interference (ICI) [2]. As a result, OMA can compensate for multipath impairments without any loss of orthogonality between subchannels. Meanwhile, for keeping orthogonal characteristics between users, the OMA scheme does not allow frequency reuse in one cell, which significantly limits the cell throughput [3]. Unlike OMA, a non-orthogonal multiple access (NOMA) technique based on the power domain can allocate a time-frequency resource to more than one user within one cell, so that the system-level throughput can be significantly enhanced in both the uplink and the downlink. Therefore, NOMA has been viewed as a promising candidate technique for future radio access.

NOMA exploits the power domain to multiplex multiple users, and signals of users are superposed in the time and frequency domains. In uplink NOMA communications, the base station (BS) receives superposed signals of users who use the same subcarrier. As different user signals go through various channels in the uplink, each signal of different users experiences distinct channel gains. As a result, the user, who has the strongest channel gain, likely has the highest received signal power. Therefore, the BS detects the user who has the strongest signal to noise ratio (SNR) first, and other nonorthogonal users act as inter-user interference (IUI). Then, an advanced signal detection technique called successive interference cancellation (SIC) [4] is implemented in a descending order of users' SNRs. In consideration of the complexity and the capacity gain, a few (two or three) simultaneous users on a subcarrier can achieve a large part of the possible gain [5]. Hence, we restrict the study in this paper to two users on a time-frequency resource, and these two users are named near user and far user according to the distances from them to the BS, i.e., the near user has a stronger SNR than the far user.

NOMA has been investigated in both the downlink and the uplink [6], and system level gains of NOMA have been demonstrated [7]. In [8], the uplink NOMA based on OFDM is studied. It has been shown that the uplink NOMA can obtain superior performance to the OFDMA in view of total system throughput and cell-edge user throughput. However, so far most research on NOMA considers time-synchronous transmissions. This assumption is reasonable in the downlink scenario, because the BS sends non-orthogonal users' signals simultaneously. The uplink users are geographically distributed in the cell, and the signals transmitted by users at different distances from the BS are received with different time delays. Besides, the transmission environment is dynamic. Therefore, the asynchronism or imperfect synchronism possibly happens in the uplink NOMA transmission [9]. In [3, 9, 10], an interference cancellation technique called triangular-SIC (TSIC) is proposed for detecting time misaligned symbols on a subcarrier in uplink NOMA transmissions, where the time asynchronism causes a symbol of one user to overlap with two symbols of another uplink NOMA user. It is noticed that the $\mathrm{CP}$ is not used to compensate for the asynchronous effects in the T-SIC process. Therefore, severe ISI and ICI may occur due to the timing offset between NOMA users (see Fig. 1 (b)). For focusing on the analysis of asynchronous multiple access interference (MAI), ISI and ICI are not considered in [3] either. Whereas, ISI and ICI may degrade the system performance severely. Therefore, we propose that the $\mathrm{CP}$ is used in the asynchronous uplink NOMA transmission for eliminating ISI \& ICI and compensating for asynchronous effects at the BS in this paper. We investigate the effects of asynchronism in the uplink NOMA transmission considering the CP for the first time in the literature, to the best of our knowledge. 
Moreover, it should be noted that asynchronous research results of OFDMA cannot be applied straightforwardly to the asynchronous uplink NOMA [10]. The asynchronism can change the probability distribution of IUI and residual interference (or error propagation) to affect negatively decoding in the NOMA system. Therefore, the new analysis of asynchronous effects in the uplink NOMA system with the CP is needed. The objective of this paper is to investigate the uplink asynchronous NOMA transmission considering CP. The contributions of this paper include:

1) Through analyzing IUI and residual interference in an asynchronous uplink NOMA scheme considering the $\mathrm{CP}$, it is shown that the asynchronous users with the conventional-SIC (Conv-SIC) have worse bit error rate (BER) performance than synchronous ones.

2) The SIC-phase compensation (SIC-PC) technique is applied to decode asynchronous far user signals. The near and far user BER performance can be improved substantially by applying the SIC-PC and the $\mathrm{CP}$, compared with using conventional-SIC (Conv-SIC) and T-SIC [3] techniques. Furthermore, the achievable rate is analyzed, where performance comparisons between SIC-PC and T-SIC are given.

The rest of this paper is organized as follows: the asynchronous uplink NOMA system model is described in Section II, the performance analysis of asynchronous uplink NOMA in Section III. Section IV provides representative simulation results. Finally, Section V presents the conclusion.

\section{ASYNCHRONOUS UPLINK NOMA SYSTEM MODEL}

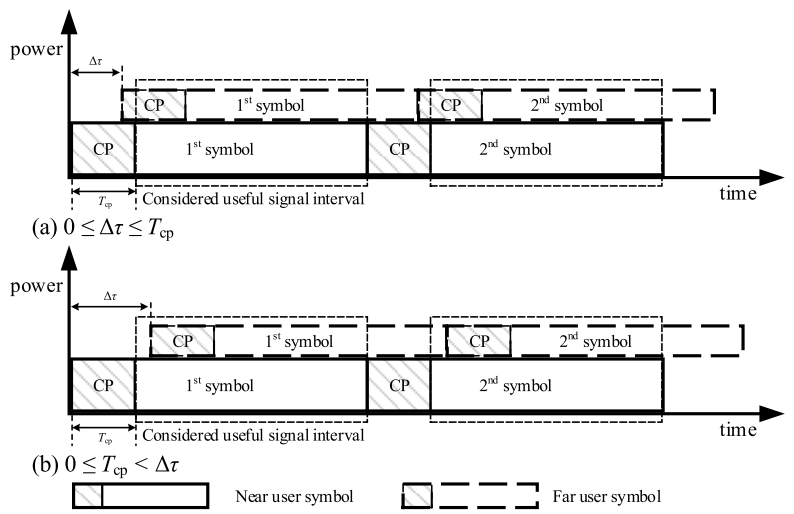

Fig. 1. Signal representation of asynchronous far user with respect to the time offset in the time domain.

The asynchronous uplink NOMA system based on OFDM is considered with a BS serving two non-orthogonal users. These two users are distributed geographically, where their distances to the BS are different and dynamic, so the asynchronous communication may occur. The received symbols from asynchronous users on the same subcarriers in the time domain are shown in Fig. 1. The relative timing difference (time offset) between the two asynchronous users is defined as $\Delta \tau, \Delta \tau \geq 0$. Considering the $\mathrm{CP}$ duration $T_{C P}$, the received signal structure can be classified into two cases with respect to the region of $\Delta \tau$, which are illustrated in Fig. 1. For concentrating on using $\mathrm{CP}$ for mitigating asynchronous effects, the multipath delay is omitted here. If the maximum multipath delay $T_{\max }$ is considered, the $\mathrm{CP}$ length will be just increased by $T_{\max }$. In Fig. 1 (a), the CP is longer than the time offset. So the considered useful signal interval only contains the first symbols of asynchronous users without any ISI and ICI. For Fig. 1 (b), ISI and ICI are introduced in the considered useful signal interval. Therefore, it is assumed that the CP is long enough for asynchronous time offset (shown as Fig. 1 (a)) in this paper, so that ISI and ICI are perfectly eliminated.

The principles of OFDM based uplink NOMA transmitter and receiver with the Conv-SIC/SIC-PC are presented in Fig. 2. It is assumed that the available bandwidth is divided into $N$ subcarriers, which are occupied simultaneously by two nonorthogonal users in one cell. As the power control is usually applied in the uplink transmission [11], the power control commands to the uplink users' transmission are assumed to be given system parameters here. We intend to analyze asynchronous effects on the system performance, so the power control strategy is beyond the scope of this paper and is not analyzed in this paper.

At the transmitter of user $k(k=1$ for the near user and $k=2$ for the far user), $X_{k, i}$ is the complex data (e.g., quadrature amplitude modulation (QAM) constellation) mapped onto the $i$ th subcarrier as an input of the $N$-point inverse fast Fourier transform (IFFT) unit. All the QAM symbols are assumed to be transmitted equally likely. The time domain signal representation of the user $k$ after IFFT is given by

$$
x_{k, n}=\frac{1}{\sqrt{N}} \sum_{i=0}^{N-1} X_{k, i} \sqrt{P_{k}} e^{j 2 \pi i n / N}, \quad 0 \leq n \leq N-1,
$$

where $x_{k, n}$ is the $n$th time sample of the user $k$, and $j$ is the imaginary unit. The average power of $X_{k, i}$ is normalized to 1, i.e., $\mathrm{E}\left[\left|X_{k, i}\right|^{2}\right]=1 . P_{k}$ is the transmit power of the user $k$, which is determined by given power control commands. $P_{1}$ is for the near user, $P_{2}$ for the far user.

In Fig. 2, the CP is preposed at each transmitted OFDM symbol before transmission. The near user and far user symbols experience timing errors due to the propagation delay between the uplink users and the BS. At the receiver, after extracting the $\mathrm{CP}$, the received signal can be expressed as

$$
y_{n}=h_{1} x_{1, n}+h_{2} \bar{x}_{2, n}+\mathfrak{n}_{n},
$$

where $h_{1}$ and $h_{2}$ are channel gains of the near user and the far user, respectively. For focusing on the effects of asynchronism, it is assumed that signals experience independent and identically distributed (i.i.d.) block Rayleigh fading $\left(P_{1}\left|h_{1}\right|^{2}>P_{2}\left|h_{2}\right|^{2}\right)$ [6]. $\mathfrak{n}_{n}$ is the additive white Gaussian noise (AWGN) with the average power $P_{A} \cdot \bar{x}_{2, n}$ is the received signal sample from the far user in the considered useful signal interval. After the fast Fourier transform (FFT) process, signals in the frequency domain are obtained, i.e., $Y_{i}=h_{1} \sqrt{P_{1}} X_{1, i}+h_{2} \sqrt{P_{2}} \bar{X}_{2, i}+\mathfrak{N}_{i}, 0 \leq i \leq N-1$. The 


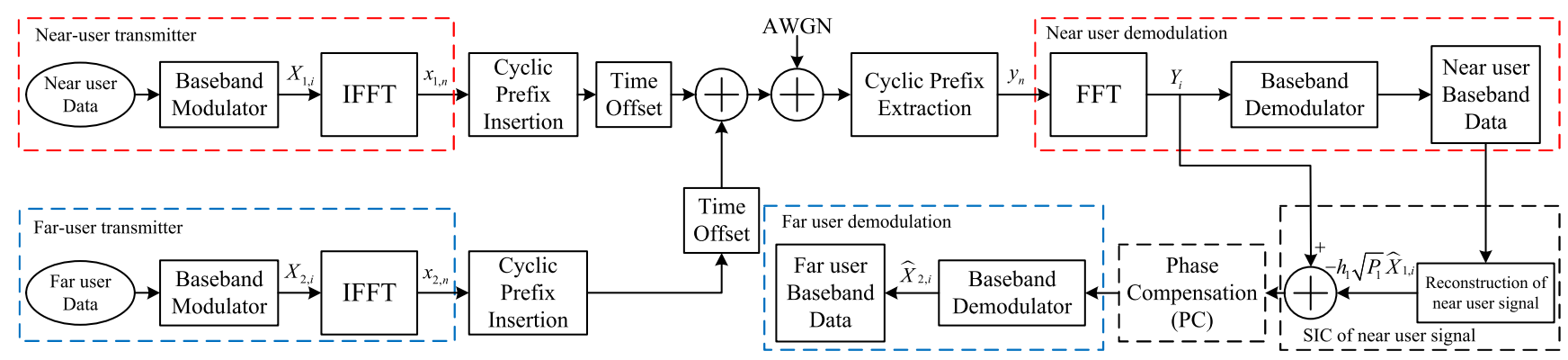

Fig. 2. Block diagram of an asynchronous uplink NOMA system.

BS decodes signals of the near user and the far user in two stages with the Conv-SIC. First, near user signals are decoded, treating signals of the far user as noise, i.e., IUI. Then, the BS subtracts the near user reconstructed signal, i.e., $h_{1} \sqrt{P_{1}} \hat{X}_{1, i}$, from the received signal. At last, the decoded far user signal $\hat{X}_{2, i}$ is obtained. For the SIC-PC technique, the difference from the Conv-SIC is that the phase compensation (PC) is added after "SIC of near user signal" in Fig. 2.

\section{Performance AnAlysis of Asynchronous UPLINK NOMA}

In this section, we are concerned with asynchronous effects on the uplink NOMA performance and the optimal PC.

\section{A. Analysis of time-asynchronous effects}

The analysis of asynchronous effects is provided in this subsection. At the receiver, $N$ discrete time samples from the considered useful signal interval can be written as

$$
\begin{aligned}
y_{\left(n+m_{1}+m_{c p}\right)}= & h_{1} x_{1, n}+h_{2} x_{2,\left(n+m_{1}-m_{2}\right)} \\
& +n_{\left(n+m_{1}+m_{c p}\right)}, \quad 0 \leq n \leq N-1,
\end{aligned}
$$

where

$$
x_{2,\left(n+m_{1}-m_{2}\right)}=\frac{1}{\sqrt{N}} \sum_{i=0}^{N-1} X_{2, i} \sqrt{P_{2}} e^{j 2 \pi i\left(n+m_{1}-m_{2}\right) / N} .
$$

$m_{1}$ and $m_{2}$ are the discrete time offsets of the near user and the far user to the reference time respectively, i.e., $\Delta \tau=$ $m_{2} T-m_{1} T$ [10], where $T$ is the sampling time interval and $0 \leq\left|m_{2}-m_{1}\right| \leq \frac{T_{C P}-T_{\max }}{T}$. Here it is assumed that $m_{1} \leq$ $m_{2}$. For $m_{1}>m_{2}$ case, the similar results below can also be derived. $m_{c p}$ is the CP length. After FFT, the frequency domain signal for decoding is given by

$$
\begin{aligned}
Y_{i}= & \frac{1}{\sqrt{N}} \sum_{n=0}^{N-1} y_{\left(n+m_{1}+m_{c p}\right)} e^{-j 2 \pi n i / N} \\
= & \sqrt{P_{1}} h_{1} X_{1, i}+\frac{1}{\sqrt{N}} \sum_{n=0}^{N-1} h_{2} x_{2,(n-m)} e^{-j 2 \pi n i / N} \\
& +\mathfrak{N}_{i}, \quad 0 \leq i \leq N-1,
\end{aligned}
$$

where $m=m_{2}-m_{1}$, and $\mathfrak{N}_{i}$ represents the frequency domain AWGN. The first term of (4) is the desired near user signal.

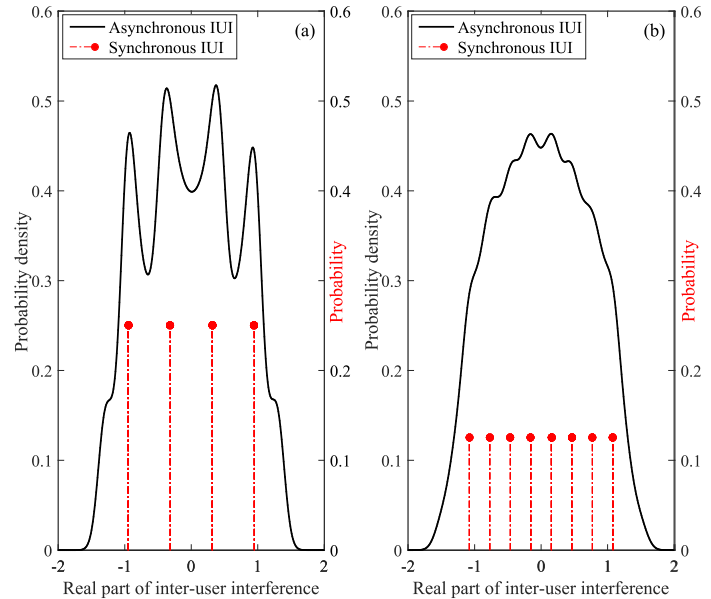

Fig. 3. Asynchronous IUI probability density and synchronous IUI probability (a) 16QAM, (b) 64QAM.

The second term represents the frequency domain IUI to the near user, given by

$$
\begin{aligned}
\eta_{2, i} & =\frac{1}{\sqrt{N}} \sum_{n=0}^{N-1} h_{2} x_{2,(n-m)} e^{-j 2 \pi n i / N} \\
& =\sum_{n=0}^{N-1} \sum_{l=0}^{N-1} \frac{1}{N} \sqrt{P_{2}} h_{2} X_{2, l} e^{j 2 \pi(n l-m l-n i) / N}
\end{aligned}
$$

If these two users are synchronized, i.e., $m_{1}=m_{2}$, (5) can be simplified as follows

$$
\eta_{2, i}=\sqrt{P_{2}} h_{2} X_{2, i}
$$

It can be seen that the synchronous IUI in the frequency domain is the constellation point from the far user. For QAM modulations, the real and imaginary parts of IUI follow the discrete uniform distribution in the synchronous case. However, in the asynchronous case, the probability density function (PDF) of IUI is changed, because the phase of IUI is interfered by the asynchronism.

Through simulations, the asynchronous IUI real part probability density (left y-axis) and the synchronous IUI real part probability (right y-axis) for 16-QAM and 64-QAM are shown in Fig. 3. The IUI average power is normalized to 1, 

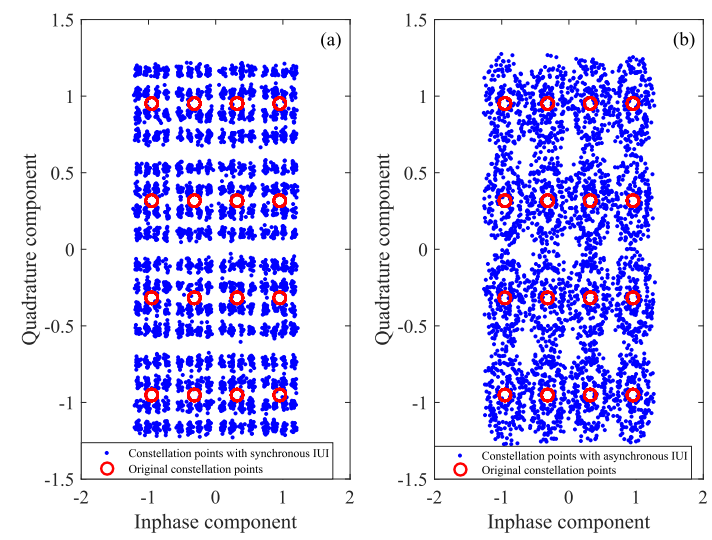

Fig. 4. Received near user 16-QAM constellation points with (a) synchronous inter-user interference (b) asynchronous inter-user interference.

$N=512$, and $m=10$. It is shown that asynchronous IUI has a wider amplitude range than synchronous IUI. Meanwhile, Fig. 4 shows the corresponding received near user constellation points in $\mathrm{SNR}_{1}=30 \mathrm{~dB}$ and $\mathrm{SNR}_{2}=17 \mathrm{~dB} . \mathrm{SNR}_{1}$ and $\mathrm{SNR}_{2}$ are the SNRs of the near user and the far user, respectively. It can be seen that the constellation points with asynchronous IUI and AWGN get mixed up with the adjacent constellation points. Therefore, the BS has a higher probability to make wrong bit decisions and to have worse BER performance for the asynchronous near user than the synchronous one, i.e., $\mathrm{BER}_{a 1}>\mathrm{BER}_{s 1}$, where the subscript "a" and "s" mean "asynchronous" and "synchronous", respectively.

Based on the decoded near user signal $\hat{X}_{1, i}$, the reconstructed near user's signal is $h_{1} \sqrt{P_{1}} \hat{X}_{1, i}$. Then, subtract the reconstructed signal from the received frequency signal $Y_{i}$ to remove the interference, given by

$$
\begin{aligned}
\tilde{Y}_{i}= & Y_{i}-h_{1} \sqrt{P_{1}} \hat{X}_{1, i} \\
= & \sum_{n=0}^{N-1} \sum_{l=0}^{N-1} \frac{1}{N} \sqrt{P_{2}} h_{2} X_{2, l} \mathrm{e}^{j 2 \pi(n l-m l-n i) / N} \\
& +\sqrt{P_{1}} h_{1}\left(X_{1, i}-\hat{X}_{1, i}\right)+\mathfrak{N}_{i}, \quad 0 \leq i \leq N-1,
\end{aligned}
$$

where $\sqrt{P_{1}} h_{1}\left(X_{1, i}-\hat{X}_{1, i}\right)$ is the residual interference for decoding the far user signal. $\sqrt{P_{2}} h_{2} X_{2, l}$ is the desired far user signal. So the signal to interference plus noise ratio (SINR) of the far user can be formulated as

$$
\operatorname{SINR}_{2}=\frac{\left|\sqrt{P_{2}} h_{2} X_{2, l}\right|^{2}}{\left|\sqrt{P_{1}} h_{1}\left(X_{1, i}-\hat{X}_{1, i}\right)\right|^{2}+\mathfrak{N}_{i}}
$$

The residual interference power has positive correlation with the near user BER [12], and $\mathrm{SINR}_{2}$ has negative correlation with the residual interference. So the residual interference has negative correlation with the near user BER. As $\mathrm{BER}_{a 1}>$ $\mathrm{BER}_{s 1}$, the asynchronous far user has lower SINR than the synchronous far user, i.e., $\operatorname{SINR}_{a 2}<\mathrm{SINR}_{s 2}$. Therefore, it can be inferred that the asynchronous far user has worse BER performance than the synchronous far user, i.e., $\mathrm{BER}_{a 2}>\mathrm{BER}_{s 2}$.
For improving the asynchronous far user BER performance, the PC is applied before decoding far user signals, as shown in Fig. 2. Suppose that the PC is $A \mathrm{e}^{j \theta}$. Hence, the Euclidean distance between the received far user constellation point with the PC and the far user original constellation point can be expressed as

$$
\begin{aligned}
& \left|A \mathrm{e}^{j \theta} \tilde{Y}_{i}-\sqrt{P_{2}} h_{2} X_{2, l}\right| \\
& =\mid \sum_{n=0}^{N-1} \sum_{l=0}^{N-1} \frac{A}{N} \sqrt{P_{2}} h_{2} X_{2, l} \mathrm{e}^{j \theta} \mathrm{e}^{j 2 \pi(n l-m l-n i) / N} \\
& \quad-\sqrt{P_{2}} h_{2} X_{2, l}+A \mathrm{e}^{j \theta} \sqrt{P_{1}} h_{1}\left(X_{1, i}-\hat{X}_{1, i}\right)+A \mathrm{e}^{j \theta} \mathfrak{N}_{i} \mid
\end{aligned}
$$

If the received far user signal has the minimum Euclidean distance to the original constellation point, the BS will have better BER performance for the far user. So the minimum Euclidean distance problem can be formed as following

$$
\min _{A, \theta}\left|A \mathrm{e}^{j \theta} \tilde{Y}_{i}-\sqrt{P_{2}} h_{2} X_{2, l}\right|
$$

subject to

$$
0<A, \quad 0 \leq \theta<2 \pi, \quad \mathrm{SNR}_{2}<\mathrm{SNR}_{1}
$$

This problem can be solved by the Levenberg-Marquardt algorithm [13]. The solution is $A=1$ and $\theta=2 \pi m i / N$.

\section{B. Achievable rate comparison}

In this subsection, the theoretical analysis from an information theoretic point of view is provided to compare the achievable rates between SIC-PC and T-SIC based NOMA systems. As we want to obtain the user achievable rate, it is assumed that IUI follows Gaussian distribution and the BS can ideally remove interference in the SIC process. As we use the CP to mitigate asynchronous effects, the longer CP is needed for the SIC-PC and Conv-SIC techniques than for the T-SIC technique. For focusing on the asynchronous effects on the user achievable rate, it is supposed that channel maximum multipath delays $T_{\max }$ of the two users are 0 . Therefore, in SIC-PC based uplink NOMA systems, the near user' shortest $\mathrm{CP}$ for mitigating asynchronous effects is $\Delta \tau$. Therefore, the users' achievable rates normalized to one unit bandwidth for the SIC-PC can be written as

$$
\left\{\begin{array}{l}
R_{P 1}=\frac{N T}{N T+\Delta \tau} \log _{2}\left(1+\frac{P_{1}\left|h_{1}\right|^{2}}{P_{2}\left|h_{2}\right|^{2}+P_{A}}\right) \\
R_{P 2}=\log _{2}\left(1+\frac{P_{2}\left|h_{2}\right|^{2}}{P_{A}}\right)
\end{array}\right.
$$

For the T-SIC technique, the CP is only used to mitigate effects of multipath signals [3]. So the shortest CP of these two users for mitigating asynchronous effects are both 0 . Achievable rates for the T-SIC are represented as

$$
\left\{\begin{array}{l}
R_{T 1}=\log _{2}\left(1+\frac{P_{1}\left|h_{1}\right|^{2}}{P_{2}\left|h_{2}\right|^{2}+P_{A}}\right) \\
R_{T 2}=\log _{2}\left(1+\frac{P_{2}\left|h_{2}\right|^{2}}{P_{A}}\right)
\end{array}\right.
$$



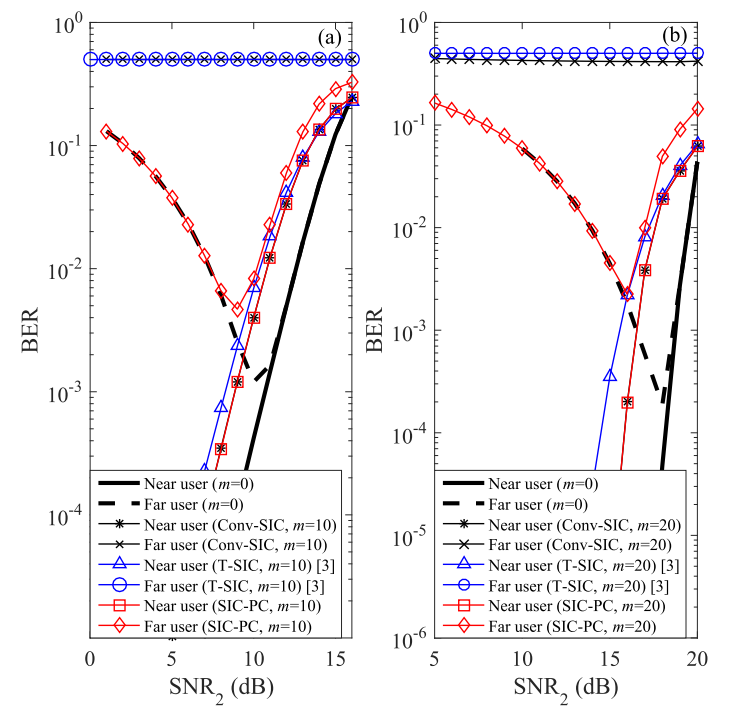

Fig. 5. QPSK and 16-QAM BER performance in the asynchronous uplink NOMA (a) QPSK in $\mathrm{SNR}_{1}=16 \mathrm{~dB}$, (b) 16-QAM in $\mathrm{SNR}_{1}=30 \mathrm{~dB}$.

From (11) and (12), it can be clearly seen that the SICPC for the far user has the same achievable rate as the TSIC. However, the SIC-PC for the near user achieves lower achievable rate than the T-SIC. As the SIC-PC and Conv-SIC use the same CP length, asynchronous uplink users with these two techniques have the same achievable rates.

\section{NUMERICAL RESUlTS}

In this section, simulation results are provided for evaluating the asynchronous uplink NOMA BER and capacity performance with different SIC techniques. The simulation environment takes a two-user uplink transmission scenario in a single-cell. It is assumed that an OFDM symbol includes 512 subcarriers, and the BS has perfect channel knowledge. All SIC processes are only the single iteration.

Fig. 5 and 6 show the uplink NOMA users' BER performance of Conv-SIC, T-SIC and SIC-PC techniques for QPSK, 16-QAM, 64-QAM and 128-QAM constellations with different time offsets $(m=0,10,20,30,40)$, where $m=0$ means that these two uplink users are synchronized. It can be easily inferred that users can obtain the same BER performance by Conv-SIC, T-SIC and SIC-PC techniques in $m=0$. In Fig.5, the near user $\mathrm{SNR}_{1}$ is set to be $16 \mathrm{~dB}$ in QPSK and $30 \mathrm{~dB}$ in 16-QAM, and the far user $\mathrm{SNR}_{2}$ is varied to investigate the BER performance. When the Conv-SIC is employed, only the near user can obtain low BER, but the asynchronous far user $(m=10,20)$ cannot achieve reliable communications. This is because of rotations of the far user constellation points as shown in (7), which degrade the far user BER performance. The asynchronous near user $(m=10,20)$ BER performance with the Conv-SIC is also degraded slightly compared with the synchronous case $(m=0)$, because the asynchronous IUI could interfere near user signals more severely than the synchronous IUI (as shown in Fig. 3 and 4).
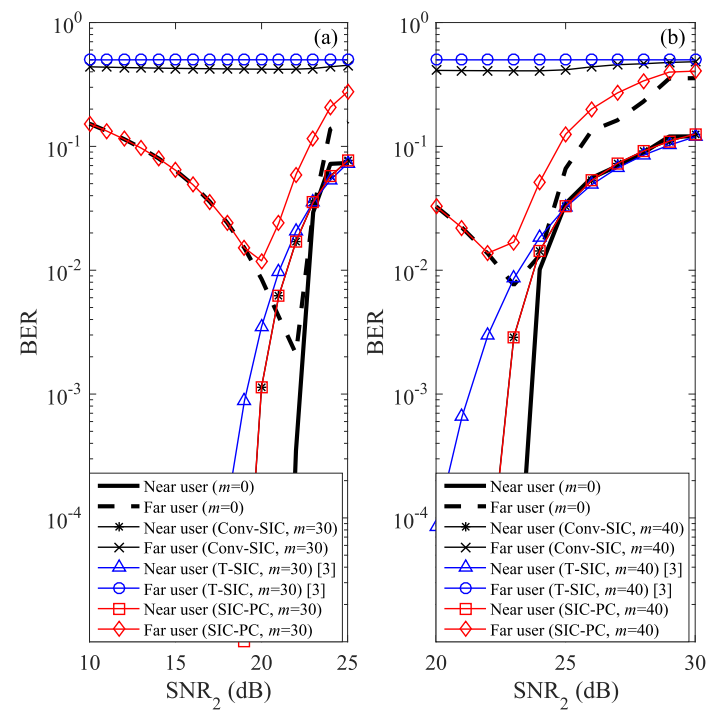

Fig. 6. 64-QAM and 128-QAM BER performance in the asynchronous uplink NOMA (a) 64-QAM in $\mathrm{SNR}_{1}=40 \mathrm{~dB}$, (b) 128-QAM in $\mathrm{SNR}_{1}=45 \mathrm{~dB}$.

In [3], the T-SIC technique is proposed to decode the asynchronous overlapped symbols. However, the T-SIC technique does not use the $\mathrm{CP}$ to compensate for the asynchronous effects. So ISI and ICI are introduced to the T-SIC decoding process due to the asynchronous time offset. Here, we are concerned with effects of ISI and ICI on the BER performance. So we propose that the Conv-SIC and SIC-PC use the long enough CP to eliminate ISI and ICI. Moreover, the PC is implemented according to (10), i.e., the received far user signals are multiplied by $e^{j 2 \pi m i / N}$ after "SIC of near user signal" process in Fig. 2. It can be seen from Fig. 5 that the asynchronous near user BER performance with the TSIC is degraded compared with the Conv-SIC and SIC-PC, because the CP length of the T-SIC technique cannot cover the asynchronous time offset. The asynchronous far user BER performance with T-SIC is degraded considerably by ISI and ICI in discrete time offsets $m=10,20$. By applying the SIC$\mathrm{PC}$ technique and the $\mathrm{CP}$ for mitigating asynchronous effects, ISI and ICI can be eliminated. Simulation results show that the near user BER performance can be increased by using the $\mathrm{CP}$ to mitigate asynchronous effects compared with the T-SIC, and the far user BER performance can be improved substantially by applying the SIC-PC, compared with ConvSIC and T-SIC techniques in the asynchronous uplink NOMA transmission. Similarly, the same conclusions can be obtained for the 64-QAM and 128-QAM constellations from Fig.6. It is also noted that the BER performance of the far user with the SIC-PC technique is still high, and error correcting codes should be used here. However, there is limited space for this paper, and the coded performance may be investigated in the future research.

Based on the analysis in Section III-B, the rate regions of asynchronous uplink NOMA systems with the SIC-PC and TSIC are plotted in Fig. 7. As the T-SIC technique does not 


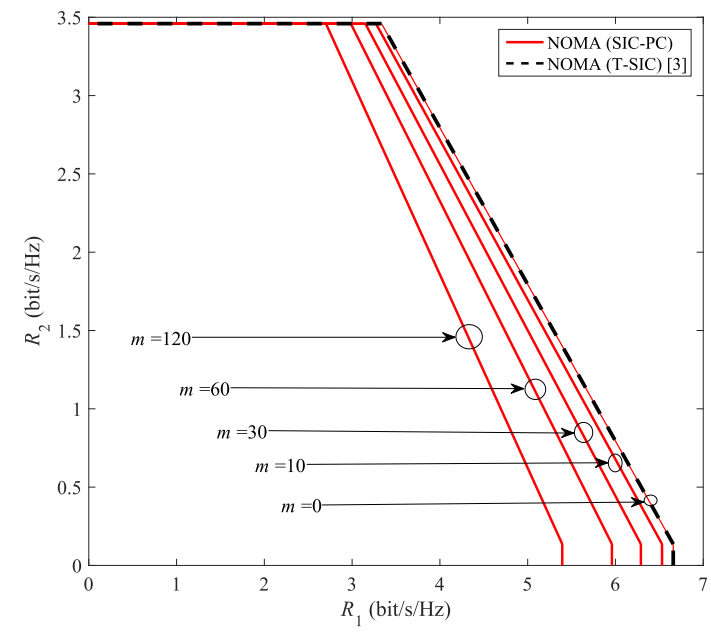

Fig. 7. Rate regions in the uplink NOMA in $\mathrm{SNR}_{1}=20 \mathrm{~dB}$ and $\mathrm{SNR}_{2}=$ $10 \mathrm{~dB}$.

use the $\mathrm{CP}$ to deal with the asynchronism, the $\mathrm{CP}$ length does not increase. The users with T-SIC can obtain the same rate region in asynchronous and synchronous transmissions. Synchronous users $(m=0)$ with SIC-PC can obtain the same region as T-SIC users. The asynchronous near user's shortest $\mathrm{CP}$ length with SIC-PC is $T_{C P}=\Delta \tau=m T$ for mitigating asynchronism. The cases of little asynchronism $(m=10,30)$ and high asynchronism $(m=60,120)$ are considered. It can be obtained that as the asynchronous time offset $\Delta \tau$ gets greater, the asynchronous near user achievable rate with SICPC gets decreased because of the longer CP. Hence, the SIC$\mathrm{PC}$ technique can be used to mitigate effects of relatively little asynchronism cases considering the achievable rate reduction.

\section{CONCLUSION}

In the asynchronous uplink NOMA transmission, different from the existing T-SIC technique [3], we propose using the $\mathrm{CP}$ to mitigate asynchronous effects in this paper. Through analyzing IUI and residual interference, it is shown that the asynchronous effects decrease the near and far user BER performance. Especially, the far user has severe BER performance degradation. The near user BER performance can be increased by using the $\mathrm{CP}$ to mitigate asynchronous effects compared with the T-SIC technique, and the asynchronous far user BER performance can be improved substantially by applying the SIC-PC, compared with the Conv-SIC and T-SIC techniques. As the $\mathrm{CP}$ is used to mitigate asynchronous effects, the near user achievable rate is reduced, which is a tradeoff between the BER performance and the achievable rate. Therefore, the CP and SIC-PC are suitable for mitigating the effects of relatively little asynchronism cases and should be considered in the practical asynchronous uplink NOMA transmission.

\section{ACKNOWLEDGMENT}

This work was supported by the National Natural Science Foundation Program of China (No.61671179), the National
Basic Research Program of China (2013CB329003), HydroQuebec, the Natural Sciences and Engineering Research Council of Canada and McGill University in the framework of the NSERC/Hydro-Quebec/McGill Industrial Research Chair in Interactive Information Infrastructure for the Power Grid (IRCPJ 406021-14), and China Scholarship Council.

\section{REFERENCES}

[1] S. Srikanth, P. A. M. Pandian, and X. Fernando, "Orthogonal frequency division multiple access in WiMAX and LTE: a comparison," IEEE Commun. Mag., vol. 50, no. 9, pp. 153-161, September 2012.

[2] L. Cimini, "Analysis and Simulation of a Digital Mobile Channel Using Orthogonal Frequency Division Multiplexing," IEEE Trans. Commun., vol. 33, no. 7, pp. 665675, Jul 1985.

[3] H. Haci, H. Zhu, and J. Wang, "Performance of Nonorthogonal Multiple Access With a Novel Asynchronous Interference Cancellation Technique," IEEE Trans. Commun., vol. 65, no. 3, pp. 1319-1335, March 2017.

[4] D. Tse and P. Viswanath, Fundamentals of wireless communication. Cambridge university press, 2005.

[5] J. Schaepperle and A. Rüegg, "Enhancement of throughput and fairness in $4 \mathrm{G}$ wireless access systems by nonorthogonal signaling," Bell Labs Techn. J., vol. 13, no. 4, p. 59, 2009.

[6] S. M. R. Islam, N. Avazov, O. A. Dobre, and K. S. Kwak, "Power-Domain Non-Orthogonal Multiple Access (NOMA) in 5G Systems: Potentials and Challenges," IEEE Commun. Surveys Tuts., vol. PP, no. 99, pp. 1-1, 2016.

[7] K. Higuchi and A. Benjebbour, "Non-orthogonal multiple access (NOMA) with successive interference cancellation for future radio access," IEICE Trans. Commun., vol. 98, no. 3, pp. 403-414, 2015.

[8] T. Takeda and K. Higuchi, "Enhanced User Fairness Using Non-Orthogonal Access with SIC in Cellular Uplink," in 2011 IEEE Vehicular Technology Conference (VTC Fall), Sept 2011, pp. 1-5.

[9] H. Haci, H. Zhu, and J. Wang, "A Novel Interference Cancellation Technique for Non-Orthogonal Multiple Access (NOMA)," in 2015 IEEE Global Communications Conference (GLOBECOM), Dec 2015, pp. 1-6.

[10] H. Haci and H. Zhu, "Performance of non-orthogonal multiple access with a novel interference cancellation method," in 2015 IEEE International Conference on Communications (ICC), June 2015, pp. 2912-2917.

[11] E. Dahlman, S. Parkvall, and J. Skold, 4G: LTE/LTEadvanced for mobile broadband. Academic press, 2013.

[12] H. Tabassum, E. Hossain, and M. J. Hossain, "Modeling and Analysis of Uplink Non-Orthogonal Multiple Access (NOMA) in Large-Scale Cellular Networks Using Poisson Cluster Processes," IEEE Trans. Commun., vol. PP, no. 99, pp. 1-1, 2017.

[13] S. J. Wright and J. Nocedal, Numerical optimization. Springer Science, 1999. 